\title{
Inequalities of the Type Hermite-Hadamard-Jensen-Mercer for Strong Convexity
}

\author{
Muhammad Adil Khan $\mathbb{D}^{1},{ }^{1}$ Saeed Anwar, ${ }^{1}$ Sadia Khalid, ${ }^{2}$ \\ and Zaid Mohammed Mohammed Mahdi Sayed (iD) ${ }^{3}$ \\ ${ }^{1}$ Department of Mathematics, University of Peshawar, Peshawar, Pakistan \\ ${ }^{2}$ Department of Mathematics, COMSATS University Islamabad, Lahore Campus, Lahore 54000, Pakistan \\ ${ }^{3}$ Department of Mathematics, University of Sáadah, Sáadah 1872, Yemen \\ Correspondence should be addressed to Zaid Mohammed Mohammed Mahdi Sayed; zaidmohamm56@gmail.com
}

Received 2 July 2021; Accepted 29 September 2021; Published 1 November 2021

Academic Editor: Erhan Set

Copyright ( ) 2021 Muhammad Adil Khan et al. This is an open access article distributed under the Creative Commons Attribution License, which permits unrestricted use, distribution, and reproduction in any medium, provided the original work is properly cited.

By using the Jensen-Mercer inequality for strongly convex functions, we present Hermite-Hadamard-Mercer inequality for strongly convex functions. Furthermore, we also present some new Hermite-Hadamard-Mercer-type inequalities for differentiable functions whose derivatives in absolute value are convex.

\section{Introduction}

Mathematical inequalities play a vital role in many fields of science. The field of mathematical inequalities and applications has enrolled an exponential improvement in the last two decades with a significant impact in other fields of modern mathematics including engineering [1], mathematical statistics [2], approximation theory [3, 4], information theory [5], and other disciplines [6]. In our work, we use Jensen-Mercer inequality for convex and strongly convex functions and also the well-known Hölder's inequality which plays a significant role in functional analysis, mathematical analysis, complex analysis, numerical analysis, statistics and probability, and partial differential equations.

First, we define convex and strongly convex functions ([7-10]) as follows:

Definition 1. A function $\vartheta: I \subset \mathbb{R} \longrightarrow \mathbb{R}$ is called convex if

$$
\vartheta(\tau m+(1-\tau) M) \leq \tau \vartheta(m)+(1-\tau) \vartheta(M),
$$

holds for all $m, M \in I$, and all $\tau \in[0,1]$.
Definition 2. A function $\vartheta: I \subset \mathbb{R} \longrightarrow \mathbb{R}$ is called strongly convex with modulus $c>0$ if

$$
\begin{aligned}
\vartheta(\tau m+(1-\tau) M) \leq & \tau \vartheta(m)+(1-\tau) \vartheta(M) \\
& -c \tau(1-\tau)(m-M)^{2},
\end{aligned}
$$

holds for all $m, M \in I$, and $\tau \in[0,1]$.

Hermite-Hadamard inequality for convex ([11]) and strongly convex functions ([12]) can be stated as follows:

Theorem 1. Let $\vartheta:[m, M] \longrightarrow \mathbb{R}$ be a convex function. Then,

$$
\vartheta\left(\frac{m+M}{2}\right) \leq \frac{1}{M-m} \int_{m}^{M} \vartheta(\tau) \mathrm{d} \tau \leq \frac{\vartheta(m)+\vartheta(M)}{2} .
$$

Theorem 2. Let $\vartheta:[m, M] \longrightarrow \mathbb{R}$ be a strongly convex function with modulus $c$. Then, 


$$
\begin{aligned}
\vartheta\left(\frac{m+M}{2}\right)+\frac{c}{12}(M-m)^{2} & \leq \frac{1}{M-m} \int_{m}^{M} \vartheta(\tau) \mathrm{d} \tau \\
& \leq \frac{\vartheta(m)+\vartheta(M)}{2}-\frac{c}{6}(M-m)^{2}
\end{aligned}
$$

For more results related to Hermite-Hadamard inequality, see $[11,13-21]$.

Jensen-Mercer inequality ([22]) for convex functions is as follows.

Theorem 3. Let $\vartheta:[m, M] \longrightarrow \mathbb{R}$ be a convex function. Then,

$$
\vartheta\left(m+M-\sum_{i=1}^{n} p_{i} x_{i}\right) \leq \vartheta(m)+\vartheta(M)-\sum_{i=1}^{n} p_{i} \vartheta\left(x_{i}\right)
$$

for each $x_{i} \in[m, M]$ and $p_{i} \in[0,1](i=1, \ldots, n)$ with $\sum_{i=1}^{n} p_{i}=1$.

First, Hermite-Hadamard-Mercer inequalities for fractional integrals was presented in [23]. For more recent studies, see [24-27].

For strongly convex functions, the Jensen-Mercer inequality is proved in [28] as follows.

Theorem 4. Let $\vartheta:[m, M] \longrightarrow \mathbb{R}$ be a strongly convex function with modulus $c$. Then,

$$
\begin{aligned}
\vartheta\left(x_{1}+x_{n}-\sum_{i=1}^{n} p_{i} x_{i}\right) \leq & \vartheta\left(x_{1}\right)+\vartheta\left(x_{n}\right)-\sum_{i=1}^{n} p_{i} \vartheta\left(x_{i}\right) \\
& -c\left(2 \sum_{i=1}^{n} p_{i} \lambda_{i}\left(1-\lambda_{i}\right)\left(x_{1}-x_{n}\right)^{2}+\sum_{i=1}^{n} p_{i}\left(x_{i}-\sum_{i=1}^{n} p_{i} x_{i}\right)^{2}\right)
\end{aligned}
$$

where $\quad \sum_{i=1}^{n} p_{i}=1, \quad \lambda_{i} \in[0,1], \quad x_{1}=\min _{1 \leq i \leq n} x_{i}$, $x_{n}=\max _{1 \leq i \leq n} x_{i}$, and $x_{i} \in[m, M]$.
Substitute $n=2$ in (6); we obtain Jensen-Mercer inequality for strongly convex functions as follows:

$$
\vartheta\left(m+M-\frac{a+b}{2}\right) \leq \vartheta(m)+\vartheta(M)-\frac{\vartheta(a)+\vartheta(b)}{2}-c\left[\left(\lambda_{1}-\lambda_{1}^{2}\right)+\left(\lambda_{2}-\lambda_{2}^{2}\right)\right](M-m)^{2}-\frac{c}{4}(a-b)^{2},
$$

where $p_{1}=p_{2}=(1 / 2), \lambda_{1}, \lambda_{2} \in[0,1]$, and $a, b \in[m, M]$.

For more details and recent results related to Theorems 3 and 4 , see [28-31].

The following definitions for fractional integrals are important as these definitions will be used throughout this study.

Definition 3. Let $\vartheta \in L[m, M]$. The Riemann-Liouville fractional integrals $\mathscr{J}_{m^{+}}^{\alpha}$ and $\mathscr{J}_{M^{-}}^{\alpha}$ of order $\alpha>0$ with $m \geq 0$ $([32,33])$ are defined by

$$
\begin{aligned}
& \mathscr{J}_{m^{+}}^{\alpha} \vartheta(x)=\frac{1}{\Gamma(\alpha)} \int_{m}^{x}(x-\tau)^{\alpha-1} \vartheta(\tau) \mathrm{d} \tau, \quad x>m, \\
& \mathscr{J}_{M^{-}}^{\alpha} \vartheta(x)=\frac{1}{\Gamma(\alpha)} \int_{x}^{M}(\tau-x)^{\alpha-1} \vartheta(\tau) \mathrm{d} \tau, \quad x<M,
\end{aligned}
$$

respectively, where $\Gamma$ is the gamma function.

\section{Main Results}

In this section, we obtain the Hermite-Hadamard-Mercer inequality for strongly convex functions by using the Jensen-Mercer inequality for strongly convex functions. We derive some new inequalities related to the right and left sides of the Hermite-Hadamard-Mercer type inequalities for differentiable functions whose derivatives in the absolute value are convex.

(8) Theorem 5. Let $\vartheta:[m, M] \longrightarrow \mathbb{R}$ be a strongly convex function with modulus $c$. Then, for all $x, z \in[m, M]$ with $x<z, m \geq 0$ and $\alpha>0$; we have 


$$
\begin{aligned}
\vartheta\left(m+M-\frac{x+z}{2}\right) \leq & \vartheta(m)+\vartheta(M)-\frac{\Gamma(\alpha+1)}{2(z-x)^{\alpha}}\left[\mathscr{J}_{x^{+}}^{\alpha} \vartheta(z)+\mathscr{J}_{z^{-}}^{\alpha} \vartheta(x)\right] \\
& -c(m-M)^{2}\left[\left(\lambda_{1}-\lambda_{1}^{2}\right)+\left(\lambda_{2}-\lambda_{2}^{2}\right)\right]-\frac{c\left(\alpha^{2}-\alpha+2\right)}{4(\alpha+1)(\alpha+2)}(z-x)^{2} \\
\leq & \vartheta(m)+\vartheta(M)-\vartheta\left(\frac{x+z}{2}\right)-c(m-M)^{2}\left[\left(\lambda_{1}-\lambda_{1}^{2}\right)+\left(\lambda_{2}-\lambda_{2}^{2}\right)\right]-\frac{c\left(\alpha^{2}-\alpha+2\right)}{2(\alpha+1)(\alpha+2)}(z-x)^{2} .
\end{aligned}
$$

Proof. By changing the variables $a=\tau x+(1-\tau) z$ and $b=$ $(1-\tau) x+\tau z$ for $x, z \in[m, M]$ and $\tau \in[0,1]$ in (7), we obtain

$$
\begin{aligned}
\vartheta\left(m+M-\frac{x+z}{2}\right) \leq & \vartheta(m)+\vartheta(M)-\frac{\vartheta(x \tau+(1-\tau) z)+\vartheta((1-\tau) x+\tau z)}{2} \\
& -c(m-M)^{2}\left[\left(\lambda_{1}-\lambda_{1}^{2}\right)+\left(\lambda_{2}-\lambda_{2}^{2}\right)\right]-\frac{c}{4}(2 \tau-1)^{2}(z-x)^{2} .
\end{aligned}
$$

Multiplying both sides of the above inequality by $\tau^{\alpha-1}$ and integrating over $[0,1]$, we get

$$
\begin{aligned}
\frac{1}{\alpha} \vartheta\left(m+M-\frac{x+z}{2}\right) \leq & \frac{1}{\alpha}[\vartheta(m)+\vartheta(M)]-\frac{1}{2} \int_{0}^{1} \tau^{\alpha-1} \vartheta(\tau x+(1-\tau) z) \mathrm{d} \tau \\
& -\frac{1}{2} \int_{0}^{1} \tau^{\alpha-1} \vartheta((1-\tau) x+\tau z) \mathrm{d} \tau-\frac{c}{\alpha}(m-M)^{2}\left[\left(\lambda_{1}-\lambda_{1}^{2}\right)+\left(\lambda_{2}-\lambda_{2}^{2}\right)\right] \\
& -\frac{c}{4}(z-x)^{2} \int_{0}^{1} \tau^{\alpha-1}(2 \tau-1)^{2} \mathrm{~d} \tau .
\end{aligned}
$$

By substituting $u=\tau x+(1-\tau) z$ and $v=(1-\tau) x+\tau z$, we have

$$
\begin{aligned}
\frac{1}{\alpha} \vartheta\left(m+M-\frac{x+z}{2}\right) \leq & \frac{1}{\alpha}[\vartheta(m)+\vartheta(M)]-\frac{1}{2(z-x)^{\alpha}}\left[\int_{x}^{z}(z-u)^{\alpha-1} \vartheta(u) \mathrm{d} u\right. \\
& \left.+\int_{x}^{z}(v-x)^{\alpha-1} \vartheta(v) \mathrm{d} v\right]-\frac{c}{\alpha}(M-m)^{2}\left[\left(\lambda_{1}-\lambda_{1}^{2}\right)+\left(\lambda_{2}-\lambda_{2}^{2}\right)\right] \\
& -\frac{c}{4}(z-x)^{2}\left[\frac{\alpha^{2}-\alpha+2}{\alpha(\alpha+1)(\alpha+2)}\right]
\end{aligned}
$$


equivalent to

$$
\begin{aligned}
\vartheta\left(m+M-\frac{x+z}{2}\right) \leq & \vartheta(m)+\vartheta(M)-\frac{\Gamma(\alpha+1)}{2(z-x)^{\alpha}}\left[\mathscr{J}_{x^{+}}^{\alpha} \vartheta(z)+\mathscr{J}_{z^{-}}^{\alpha} \vartheta(x)\right] \\
& -c(M-m)^{2}\left[\left(\lambda_{1}-\lambda_{1}^{2}\right)+\left(\lambda_{2}-\lambda_{2}^{2}\right)\right]-\frac{c\left(\alpha^{2}-\alpha+2\right)}{4(\alpha+1)(\alpha+2)}(z-x)^{2},
\end{aligned}
$$

which is the left side of (9).

To obtain the right side of (9), use the definition of strongly convex function $\vartheta$, and we have

$$
\vartheta\left(\frac{x+z}{2}\right)=\vartheta\left(\frac{\tau x+(1-\tau) z+\tau z+(1-\tau) x}{2}\right)
$$

$$
\begin{aligned}
\leq & \frac{\vartheta(\tau x+(1-\tau) z)}{2}+\frac{\vartheta((1-\tau) x+\tau z)}{2} \\
& -\frac{c}{4}(2 \tau-1)^{2}(z-x)^{2} .
\end{aligned}
$$

Multiplying both sides of the above inequality by $\tau^{\alpha-1}$ and integrating over $[0,1]$, we have

$$
\begin{aligned}
\frac{1}{\alpha} \vartheta\left(\frac{x+z}{2}\right) \leq & \frac{1}{2} \int_{0}^{1} \tau^{\alpha-1} \vartheta(\tau x+(1-\tau) z) \mathrm{d} \tau+\frac{1}{2} \int_{0}^{1} \tau^{\alpha-1} \vartheta((1-\tau) x+\tau z) \mathrm{d} \tau \\
& -\frac{c}{4}(z-x)^{2} \int_{0}^{1} \tau^{\alpha-1}(2 \tau-1)^{2} \mathrm{~d} \tau
\end{aligned}
$$

By substituting $u=\tau x+(1-\tau) z$ and $v=(1-\tau) x+\tau z$, we obtain

$$
\begin{aligned}
\frac{1}{\alpha} \vartheta\left(\frac{x+z}{2}\right) \leq & \frac{1}{2(z-x)^{\alpha}} \int_{x}^{z}(z-u)^{\alpha-1} \vartheta(u) \mathrm{d} u+\frac{1}{2(z-x)^{\alpha}} \int_{x}^{z}(v-x)^{\alpha-1} \vartheta(v) \mathrm{d} v \\
& -\frac{c}{4}(z-x)^{2}\left[\frac{\alpha^{2}-\alpha+2}{\alpha(\alpha+1)(\alpha+2)}\right] \\
\Rightarrow & \vartheta\left(\frac{x+z}{2}\right) \leq \frac{\Gamma(\alpha+1)}{2(z-x)^{\alpha}}\left[\mathscr{F}_{x^{+}}^{\alpha} \vartheta(z)+\mathscr{J}_{z^{-}}^{\alpha} \vartheta(x)\right]-\frac{c\left(\alpha^{2}-\alpha+2\right)}{4(\alpha+1)(\alpha+2)}(z-x)^{2}
\end{aligned}
$$

From the above expression, we can write

$$
\begin{aligned}
& \vartheta(m)+\vartheta(M)-\frac{\Gamma(\alpha+1)}{2(z-x)^{\alpha}}\left[\mathscr{J}_{x^{+}}^{\alpha} \vartheta(z)+\mathscr{J}_{z^{-}}^{\alpha} \vartheta(x)\right]-\frac{c\left(\alpha^{2}-\alpha+2\right)}{4(\alpha+1)(\alpha+2)}(z-x)^{2} \\
& \quad-c(M-m)^{2}\left[\left(\lambda_{1}-\lambda_{1}^{2}\right)+\left(\lambda_{2}-\lambda_{2}^{2}\right)\right] \\
& \leq \vartheta(m)+\vartheta(M)-\vartheta\left(\frac{x+z}{2}\right)-\frac{c\left(\alpha^{2}-\alpha+2\right)}{2(\alpha+1)(\alpha+2)}(z-x)^{2} \\
& \quad-c(m-M)^{2}\left[\left(\lambda_{1}-\lambda_{1}^{2}\right)+\left(\lambda_{2}-\lambda_{2}^{2}\right)\right] .
\end{aligned}
$$




$$
\begin{aligned}
\vartheta\left(m+M-\frac{x+z}{2}\right) \leq & \frac{\Gamma(\alpha+1)}{2(z-x)^{\alpha}}\left[\mathscr{J}_{(m+M-x)^{-}}^{\alpha} \vartheta(m+M-z)+\mathscr{J}_{(m+M-z)^{+}}^{\alpha} \vartheta(m+M-x)\right] \\
& -\frac{c\left(\alpha^{2}-\alpha+2\right)}{4(\alpha+1)(\alpha+2)}(z-x)^{2} \\
\leq & \frac{\vartheta(m+M-x)+\vartheta(m+M-z)}{2}-\frac{c}{4}(z-x)^{2} \\
\leq & \vartheta(m)+\vartheta(M)-\frac{\vartheta(x)+\vartheta(z)}{2}-\frac{c}{4}(z-x)^{2} .
\end{aligned}
$$

Proof. Since $\vartheta$ is strongly convex, we can write

$$
\begin{aligned}
\vartheta\left(m+M-\frac{a+b}{2}\right)= & \vartheta\left(\frac{m+M-a+m+M-b}{2}\right) \\
\leq & \frac{1}{2}[\vartheta(m+M-a) \\
& +\vartheta(m+M-b)]-\frac{c}{4}(b-a)^{2} .
\end{aligned}
$$

By changing the variables $m+M-a=\tau(m+M-x)+$ $(1-\tau)(m+M-z)$ and $m+M-b=(1-\tau)(m+M-x)$ $+\tau(m+M-z)$, such that $\tau \in[0,1]$, we obtain

$$
\begin{aligned}
\vartheta\left(m+M-\frac{x+z}{2}\right) \leq & \frac{1}{2} \vartheta(\tau(m+M-x)+(1-\tau)(m+M-z)) \\
& +\frac{1}{2} \vartheta((1-\tau)(m+M-x)+\tau(m+M-z))-\frac{c}{4}\left[(2 \tau-1)^{2}(z-x)^{2}\right] .
\end{aligned}
$$

Multiplying both sides by $\tau^{\alpha-1}$, integrating over $[0,1]$ and in the obtained expression by substituting $u=\tau(m+$ $M-x)+(1-\tau)(m+M-z)$ and $v=(1-\tau)(m+M-x)$ $+\tau(m+M-z)$, we get the left side of (18).
Using the strongly convex function $\vartheta$ and the Jensen-Mercer inequality, we obtain

$$
\begin{aligned}
& \vartheta(\tau(m+M-x)+(1-\tau)(m+M-z))+\vartheta((1-\tau)(m+M-x)+\tau(m+M-z)) \\
& \leq \vartheta(m+M-x)+\vartheta\left((m+M-z)-2 c\left(\tau-\tau^{2}\right)(z-x)^{2}\right. \\
& \leq 2[\vartheta(m)+\vartheta(M)]-[\vartheta(x)+\vartheta(z)]-2 c\left(\tau-\tau^{2}\right)(z-x)^{2} .
\end{aligned}
$$

Multiplying both sides of the above inequality by $\tau^{\alpha-1}$, integrating over $[0,1]$ and in the obtained expression by 
substituting $u=\tau(m+M-x)+(1-\tau)(m+M-z)$ and $v=(1-\tau)(m+M-x)+\tau(m+M-z)$, we get the right side of (18).
Corollary 1. If we take $\alpha=1$ in Theorem 5 , then we have

$$
\begin{aligned}
\vartheta\left(M+m-\frac{x+z}{2}\right) \leq & \vartheta(m)+\vartheta(M)-\int_{0}^{1} \vartheta(\tau x+(1-\tau) z) \mathrm{d} \tau \\
& -c(M-m)^{2}\left[\left(\lambda_{1}-\lambda_{1}^{2}\right)+\left(\lambda_{2}-\lambda_{2}^{2}\right)\right]-\frac{c}{12}(z-x)^{2} \\
\leq & \vartheta(m)+\vartheta(M)-\vartheta\left(\frac{x+z}{2}\right)-c(M-m)^{2}\left[\left(\lambda_{1}-\lambda_{1}^{2}\right)+\left(\lambda_{2}-\lambda_{2}^{2}\right)\right]-\frac{c}{6}(z-x)^{2} .
\end{aligned}
$$

Corollary 2. If we take $\alpha=1$ in Theorem 6 , then we have

$$
\begin{aligned}
\vartheta\left(m+M-\frac{x+z}{2}\right) & \leq \frac{1}{z-x} \int_{x}^{z} \vartheta(m+M-\tau) \mathrm{d} \tau-\frac{c}{12}(z-x)^{2} \\
& \leq \vartheta(m)+\vartheta(M)-\frac{\vartheta(x)+\vartheta(z)}{2}-\frac{c}{4}(z-x)^{2} .
\end{aligned}
$$

Remark 1. If we take $x=m$ and $z=M$ in (23), we obtain the Hermite-Hadamard inequality for strongly convex function given in [12].

Theorem 7. Let all the assumptions of Theorem 5 hold. Then,

$$
\begin{aligned}
\vartheta\left(m+M-\frac{x+z}{2}\right) \leq & \frac{2^{\alpha-1} \Gamma(\alpha+1)}{(z-x)^{\alpha}}\left[\mathscr{f}_{(m+M-((x+z) / 2))^{-}}^{\alpha} \vartheta(m+M-z)\right. \\
& \left.+\mathscr{f}_{(m+M-((x+z) / 2))^{+}}^{\alpha} \vartheta(m+M-x)\right]-\frac{c}{2(\alpha+1)(\alpha+2)}(z-x)^{2} \\
& \leq \vartheta(m)+\vartheta(M)-\frac{\vartheta(x)+\vartheta(z)}{2}-c\left[\left(\lambda_{1}-\lambda_{1}^{2}\right)+\left(\lambda_{2}-\lambda_{2}^{2}\right)\right](m-M)^{2}-\frac{c}{4}(z-x)^{2} .
\end{aligned}
$$

Proof. Since $\vartheta$ is a strongly convex function, we can write

$$
\begin{aligned}
\vartheta\left(m+M-\frac{a+b}{2}\right) & =\vartheta\left(\frac{m+M-a+m+M-b}{2}\right) \\
& \leq \frac{\vartheta(m+M-a)}{2}+\frac{\vartheta(m+M-b)}{2}-\frac{c}{4}(a-b)^{2} .
\end{aligned}
$$

$$
\begin{aligned}
\vartheta\left(m+M-\frac{x+y}{2}\right) \leq & \frac{\vartheta(m+M-((\tau / 2) x+((2-\tau) / 2)) z)}{2}+\frac{\vartheta(m+M-(((2-\tau) / 2) x+(\tau / 2)) z)}{2} \\
& -\frac{c}{4}(1-\tau)^{2}(z-x)^{2} .
\end{aligned}
$$

Multiplying both sides of the above inequality by $\tau^{\alpha-1}$, integrating over $[0,1]$ and in the obtained expression by
Taking $a=(\tau / 2) x+((2-\tau) / 2) z$ and $b=((2-\tau) / 2) x+$ $(\tau / 2) z$ such that $x, z \in[m, M]$ and $\tau \in[0,1]$, we obtain substituting $u=m+M-((\tau / 2) x+((2-\tau) / 2) z)$ and $v=m$ $+M-(((2-\tau) / 2) x+(\tau / 2) z)$, we get the first inequality of $(24)$. 
For obtaining the second inequality of (24), use the Jensen-Mercer inequality for strongly convex function, and we get

$$
\begin{aligned}
\vartheta\left(m+M-\left(\frac{\tau}{2} x+\frac{2-\tau}{2} z\right)\right) \leq & \vartheta(m)+\vartheta(M)-\left[\frac{\tau}{2} \vartheta(x)+\frac{2-\tau}{2} \vartheta(z)\right] \\
& -c\left[\left(\lambda_{1}-\lambda_{1}^{2}\right)+\left(\lambda_{2}-\lambda_{2}^{2}\right)\right](m-M)^{2}-c\left(\frac{\tau}{2}\right)\left(1-\frac{\tau}{2}\right)(z-x)^{2}, \\
\vartheta\left(m+M-\left(\frac{2-\tau}{2} x+\frac{\tau}{2} z\right)\right) \leq & \vartheta(m)+\vartheta(M)-\left[\frac{2-\tau}{2} \vartheta(x)+\frac{\tau}{2} \vartheta(z)\right] \\
& -c\left[\left(\lambda_{1}-\lambda_{1}^{2}\right)+\left(\lambda_{2}-\lambda_{2}^{2}\right)\right](m-M)^{2}-c\left(\frac{\tau}{2}\right)\left(1-\frac{\tau}{2}\right)(z-x)^{2} .
\end{aligned}
$$

Adding these inequalities, multiplying both sides by $\tau^{\alpha-1}$, integrating over $[0,1]$ and in the obtained expression by substituting $u=m+M-((\tau / 2) x+((2-\tau) / 2) / 2) z)$ and
$v=m+M-((2-\tau) / 2) x+(\tau / 2) z)$, we get the second inequality of (24).

Theorem 8. Let all the assumptions of Theorem 5 hold. Then,

$$
\begin{aligned}
\vartheta\left(m+M-\frac{x+z}{2}\right) \leq & \vartheta(m)+\vartheta(M)-\frac{2^{\alpha-1} \Gamma(\alpha+1)}{(z-x)^{\alpha}}\left[\mathscr{J}_{((x+z) / 2)^{-}}^{\alpha} \vartheta(x)+\mathscr{J}_{((x+z) / 2)^{+}}^{\alpha} \vartheta(z)\right] \\
& -c\left[\left(\lambda_{1}-\lambda_{1}^{2}\right)+\left(\lambda_{2}-\lambda_{2}^{2}\right)\right](m-M)^{2}-\frac{c}{2(\alpha+1)(\alpha+2)}(z-x)^{2} \\
\leq & \vartheta(m)+\vartheta(M)-\vartheta\left(\frac{x+z}{2}\right)-c\left[\left(\lambda_{1}-\lambda_{1}^{2}\right)+\left(\lambda_{2}-\lambda_{2}^{2}\right)\right](m-M)^{2} \\
& -\frac{c}{(\alpha+1)(\alpha+2)}(z-x)^{2} .
\end{aligned}
$$

Proof. Using the Jensen-Mercer inequality for strongly convex function, we have

$$
\vartheta\left(m+M-\frac{a+b}{2}\right) \leq \vartheta(m)+\vartheta(M)-\frac{\vartheta(a)+\vartheta(b)}{2}-c\left[\left(\lambda_{1}-\lambda_{1}^{2}\right)+\left(\lambda_{2}-\lambda_{2}^{2}\right)\right](m-M)^{2}-\frac{c}{4}(a-b)^{2} .
$$

By writing $a=(\tau / 2) x+((2-\tau) / 2) z$ and $b=((2-\tau) / 2)$ $x+(\tau / 2) z$, such that $x, z \in[m, M]$ and $\tau \in[0,1]$, we obtain

$$
\begin{aligned}
\vartheta\left(m+M-\frac{x+z}{2}\right) \leq & \vartheta(m)+\vartheta(M)-\frac{\vartheta((\tau / 2) x+((2-\tau) / 2) z)+\vartheta(((2-\tau) / 2) x+(\tau / 2) z)}{2} \\
& -c\left[\left(\lambda_{1}-\lambda_{1}^{2}\right)+\left(\lambda_{2}-\lambda_{2}^{2}\right)\right](m-M)^{2}-\frac{c}{4}(1-\tau)^{2}(z-x)^{2} .
\end{aligned}
$$

Multiplying both sides of the above inequality by $\tau^{\alpha-1}$, integrating over $[0,1]$ and in the obtained expression by substituting $u=(\tau / 2) x+((2-\tau) / 2) z$ and $v=((2-\tau) / 2) x$ $+(\tau / 2) z$, we get the left side of $(28)$. 
For obtaining the right side of (28), use the definition of strongly convex function, and we have

$$
\vartheta\left(\frac{a+b}{2}\right) \leq \frac{1}{2} \vartheta(a)+\frac{1}{2} \vartheta(b)-\frac{c}{4}(a-b)^{2} .
$$

By writing $a=(\tau / 2) x+((2-\tau) / 2) z \quad$ and $b=((2-\tau) / 2) x+(\tau / 2) z$, such that $x, z \in[m, M]$ and $\tau \in[0,1]$, we obtain

$$
\begin{aligned}
\vartheta\left(\frac{x+z}{2}\right) \leq & \frac{1}{2}\left[\vartheta\left(\frac{\tau}{2} x+\frac{2-\tau}{2} z\right)+\vartheta\left(\frac{2-\tau}{2} x+\frac{\tau}{2} z\right)\right] \\
& -\frac{c}{4}(z-x)^{2}(1-\tau)^{2} .
\end{aligned}
$$

Multiplying both sides of the above inequality by $\tau^{\alpha-1}$, integrating over $[0,1]$ and substituting $u=(\tau / 2) x+((2-$ $\tau) / 2) z$ and $v=((2-\tau) / 2) x+(\tau / 2) z$, we get

$$
\begin{aligned}
\vartheta\left(\frac{x+z}{2}\right) \leq & \frac{2^{\alpha-1} \Gamma(\alpha+1)}{(z-x)^{\alpha}}\left[\mathscr{F}_{((x+z) / 2)^{+}}^{\alpha} \vartheta(z)+\mathscr{J}_{((x+z) / 2)^{-}}^{\alpha} \vartheta(x)\right] \\
& -\frac{c}{2(\alpha+1)(\alpha+2)}(z-x)^{2} .
\end{aligned}
$$

From the above expression, we obtain the right side of (28).

Lemma 1. Let $\vartheta:[m, M] \longrightarrow \mathbb{R}$ be a differentiable function, such that $\vartheta^{\prime} \in L[m, M]$. Then, for all $x, z \in[m, M]$, such that $x<z, \alpha>0$ and $\tau \in[0,1]$, we have

$$
\begin{aligned}
& \frac{\vartheta(m+M-x)+\vartheta(m+M-z)}{2}-\frac{\Gamma(\alpha+1)}{2(z-x)^{\alpha}}\left[\mathscr{J}_{(m+M-z)^{+}}^{\alpha} \vartheta(m+M-x)\right. \\
& \left.\quad+\mathscr{J}_{(m+M-x)^{-}}^{\alpha} \vartheta(m+M-z)\right]-\frac{\alpha c}{(\alpha+1)(\alpha+2)}(z-x)^{2} \\
& =\frac{z-x}{2}\left\{\int_{0}^{1}\left[\left(\tau^{\alpha}-(1-\tau)^{\alpha}\right) \vartheta^{\prime}(m+M-(\tau x+(1-\tau) z))-2 \alpha c(z-x)\left(\tau^{\alpha}-\tau^{\alpha+1}\right)\right] \mathrm{d} \tau\right\} .
\end{aligned}
$$

Proof. We can write

$$
\begin{aligned}
I= & \int_{0}^{1}\left[\left(\tau^{\alpha}-(1-\tau)^{\alpha}\right) \vartheta^{\prime}(m+M-(\tau x+(1-\tau) z))-2 \alpha c(z-x)\left(\tau^{\alpha}-\tau^{\alpha+1}\right)\right] \mathrm{d} \tau \\
= & \int_{0}^{1} \tau^{\alpha} \vartheta^{\prime}(m+M-(\tau x+(1-\tau) z)) \mathrm{d} \tau-\int_{0}^{1}(1-\tau)^{\alpha} \vartheta^{\prime}(m+M-(\tau x+(1-\tau) z)) \mathrm{d} \tau \\
& -2 \alpha c(z-x) \int_{0}^{1}\left(\tau^{\alpha}-\tau^{\alpha+1}\right) \mathrm{d} \tau,
\end{aligned}
$$

that is,

Integrating each term, we obtain

$$
I=I_{1}-I_{2}-2 \alpha c(z-x) I_{3} .
$$

$$
\begin{aligned}
I_{1} & =\int_{0}^{1} \tau^{\alpha} \vartheta^{\prime}(m+M-(\tau x+(1-\tau) z)) \mathrm{d} \tau \\
& =\left.\frac{\tau^{\alpha} \vartheta(m+M-(\tau x+(1-\tau) z))}{z-x}\right|_{0} ^{1}-\frac{\alpha}{z-x} \int_{0}^{1} \tau^{\alpha-1} \vartheta(m+M-(\tau x+(1-\tau) z)) \mathrm{d} \tau \\
& =\frac{\vartheta(m+M-x)}{z-x}-\frac{\Gamma(\alpha+1)}{(z-x)^{\alpha+1}} \mathcal{J}_{(m+M-x)^{-}}^{\alpha} \vartheta(m+M-z),
\end{aligned}
$$




$$
\begin{aligned}
I_{2} & =\int_{0}^{1}(1-\tau)^{\alpha} \vartheta^{\prime}(m+M-(\tau x+(1-\tau) z)) \mathrm{d} \tau \\
& =\frac{(1-\tau)^{\alpha} \vartheta(m+M-(\tau x+(1-\tau) z))_{0}^{1}+\frac{\alpha}{z-x} \int_{0}^{1}(1-\tau)^{\alpha-1} \vartheta(m+M-(\tau x+(1-\tau) z)) \mathrm{d} \tau}{z-x}-\frac{\Gamma(\alpha+1)}{(z-x)^{\alpha+1}} \mathscr{J}_{(m+M-z)^{+}}^{\alpha} \vartheta(m+M-x), \\
& =-\frac{1(m+M-x)}{z-x}-\frac{1}{(\alpha+1)(\alpha+2)}
\end{aligned}
$$

Substituting the values of $I_{1}, I_{2}$, and $I_{3}$ in (36) and simplifying, we obtain

$$
\begin{aligned}
I= & \frac{\vartheta(m+M-x)+\vartheta(m+M-z)}{z-x}-\frac{\Gamma(\alpha+1)}{(z-x)^{\alpha+1}}\left[\mathscr{J}_{(m+M-z)^{+}}^{\alpha} \vartheta(m+M-x)\right. \\
& \left.+\mathscr{J}_{(m+M-x)^{-}}^{\alpha} \vartheta(m+M-z)\right]-\frac{2 \alpha c}{(\alpha+1)(\alpha+2)}(z-x) .
\end{aligned}
$$

Now, multiplying both sides by $((z-x) / 2)$, we get Corollary 3 . If we take $\alpha=1$ in Lemma 1 , then we obtain (34).

$$
\begin{aligned}
& \frac{\vartheta(m+M-x)+\vartheta(m+M-z)}{2}-\frac{1}{z-x} \int_{m+M-z}^{m+M-x} \vartheta(u) \mathrm{d} u-\frac{c}{6}(z-x)^{2} \\
& =\frac{z-x}{2}\left[\int_{0}^{1}\left[(2 \tau-1) \vartheta^{\prime}(m+M-(\tau x+(1-\tau) z))-2 c(z-x)\left(\tau-\tau^{2}\right)\right] \mathrm{d} \tau\right] .
\end{aligned}
$$

Lemma 2. Let all the assumptions of Lemma 1 hold. Then,

$$
\begin{aligned}
& \frac{2^{\alpha-1} \Gamma(\alpha+1)}{(z-x)^{\alpha}}\left[\mathcal{J}_{(m+M-((x+z) / 2))^{+}}^{\alpha} \vartheta(m+M-x)+\mathcal{J}_{(m+M-((x+z) / 2))^{-}}^{\alpha} \vartheta(m+M-z)\right] \\
& \quad-\vartheta\left(m+M-\frac{x+z}{2}\right)-\frac{c}{2(\alpha+1)(\alpha+2)}(z-x)^{2} \\
& =\frac{z-x}{4} \int_{0}^{1} \tau^{\alpha}\left[\vartheta^{\prime}\left(m+M-\left(\frac{2-\tau}{2} x+\frac{\tau}{2} z\right)\right)\right. \\
& \left.\quad-\vartheta^{\prime}\left(m+M-\left(\frac{\tau}{2} x+\frac{2-\tau}{2} z\right)\right)-2 c(z-x)(1-\tau)\right] \mathrm{d} \tau .
\end{aligned}
$$


Proof. We can write

$$
\begin{aligned}
& \frac{z-x}{4} \int_{0}^{1} \tau^{\alpha}\left[\vartheta^{\prime}\left(m+M-\left(\frac{2-\tau}{2} x+\frac{\tau}{2} z\right)\right)\right. \\
& \left.-\vartheta^{\prime}\left(m+M-\left(\frac{\tau}{2} x+\frac{2-\tau}{2} z\right)\right)-2 c(z-x)(1-\tau)\right] \mathrm{d} \tau \\
& =\frac{z-x}{4}\left\{I_{1}-I_{2}-2 c(z-x) I_{3}\right\} .
\end{aligned}
$$

Simplifying each term separately, we obtain

$$
I_{1}=\int_{0}^{1} \tau^{\alpha} \vartheta^{\prime}\left(m+M-\left(\frac{2-\tau}{2} x+\frac{\tau}{2} z\right)\right) \mathrm{d} \tau .
$$

Using integration by parts and substitution, we get

$$
\begin{aligned}
I_{1}= & \frac{2}{x-z} \vartheta\left(m+M-\frac{x+z}{2}\right) \\
& +\frac{2^{\alpha+1} \Gamma(\alpha+1)}{(z-x)^{\alpha+1}} \mathcal{J}_{(m+M-((x+z) / 2))^{+}}^{\alpha} \vartheta(m+M-x) .
\end{aligned}
$$

We have

$$
I_{2}=\int_{0}^{1} \tau^{\alpha} \vartheta^{\prime}\left(m+M-\left(\frac{\tau}{2} x+\frac{2-\tau}{2} z\right)\right) \mathrm{d} \tau .
$$

Using integration by parts and substitution, we get

$$
\begin{aligned}
I_{2}= & \frac{2}{z-x} \vartheta\left(m+M-\frac{x+z}{2}\right) \\
& +\frac{2^{\alpha+1} \Gamma(\alpha+1)}{(z-x)^{\alpha+1}} \mathcal{J}_{(m+M-((x+z) / 2))^{-}}^{\alpha} \vartheta(m+M-z) .
\end{aligned}
$$

Similarly, we have

$$
I_{3}=\frac{1}{(\alpha+1)(\alpha+2)}
$$

Using (43)-(46) in (41), we obtain (40).

Theorem 9. Let $\vartheta:[m, M] \longrightarrow \mathbb{R}$ be a differentiable function, such that $\left|\vartheta^{\prime}\right|$ is convex on $[m, M]$. Then, for all $x, z \in[m, M]$ with $x<z$ and $\alpha>0$, we have

$$
\begin{aligned}
& \mid \frac{\vartheta(m+M-x)+\vartheta(m+M-z)}{2}-\frac{\Gamma(\alpha+1)}{2(z-x)^{\alpha}}\left[\mathscr{f}_{(m+M-z)^{+}}^{\alpha} \vartheta(m+M-x)\right. \\
& \left.\quad+\mathscr{J}_{(m+M-x)^{-}}^{\alpha} \vartheta(m+M-z)\right]-\frac{\alpha c}{(\alpha+1)(\alpha+2)}(z-x)^{2} \mid \\
& \leq \frac{z-x}{\alpha+1}\left(1-\frac{1}{2^{\alpha}}\right)\left[\left|\vartheta^{\prime}(M)\right|+\left|\vartheta^{\prime}(m)\right|-\frac{\left|\vartheta^{\prime}(x)\right|+\left|\vartheta^{\prime}(z)\right|}{2}+\frac{2^{\alpha} \alpha c(z-x)}{\left(2^{\alpha}-1\right)(\alpha+2)}\right] .
\end{aligned}
$$

Proof. Using Lemma 1, we can write

$$
\begin{aligned}
& \mid \frac{\vartheta(m+M-x)+\vartheta(m+M-z)}{2}-\frac{\Gamma(\alpha+1)}{2(z-x)^{\alpha}}\left[\mathscr{J}_{(m+M-z)^{+}}^{\alpha} \vartheta(m+M-x)\right. \\
& \left.\quad+\mathcal{J}_{(m+M-x)^{-}}^{\alpha} \vartheta(m+M-z)\right]-\frac{\alpha c}{(\alpha+1)(\alpha+2)}(z-x)^{2} \mid \\
& \leq \frac{z-x}{2}\left\{\int_{0}^{1}\left[\left|\tau^{\alpha}-(1-\tau)^{\alpha}\right|\left|\vartheta^{\prime}(m+M-(\tau x+(1-\tau) z))\right|+\left|-2 \alpha c(z-x)\left(\tau^{\alpha}-\tau^{\alpha+1}\right)\right|\right] \mathrm{d} \tau\right\} \\
& =\frac{z-x}{2}\left\{\int_{0}^{1}\left[\left|\tau^{\alpha}-(1-\tau)^{\alpha}\right|\left|\vartheta^{\prime}(m+M-(\tau x+(1-\tau) z))\right|+2 \alpha c(z-x)\left|\tau^{\alpha}-\tau^{\alpha+1}\right|\right] \mathrm{d} \tau\right\} \\
& \leq \frac{z-x}{2}\left\{\int_{0}^{1}\left|\tau^{\alpha}-(1-\tau)^{\alpha}\right|\left[\left|\vartheta^{\prime}(m)\right|+\left|\vartheta^{\prime}(M)\right|-\left(t\left|\vartheta^{\prime}(x)\right|+(1-\tau)\left|\vartheta^{\prime}(z)\right|\right)\right] \mathrm{d} \tau\right. \\
& \left.\quad+2 \alpha c(z-x) \int_{0}^{1}\left|\tau^{\alpha}-\tau^{\alpha+1}\right| \mathrm{d} \tau\right\}
\end{aligned}
$$




$$
\begin{aligned}
= & \frac{z-x}{2} \int_{0}^{1 / 2}\left((1-\tau)^{\alpha}-\tau^{\alpha}\right)\left[\left|\vartheta^{\prime}(m)\right|+\left|\vartheta^{\prime}(M)\right|-\left(\tau\left|\vartheta^{\prime}(x)\right|+(1-\tau)\left|\vartheta^{\prime}(z)\right|\right)\right] \mathrm{d} \tau \\
& +\int_{1 / 2}^{1}\left(\tau^{\alpha}-(1-\tau)^{\alpha}\right)\left[\left|\vartheta^{\prime}(m)\right|+\left|\vartheta^{\prime}(M)\right|-\left(\tau\left|\vartheta^{\prime}(x)\right|+(1-\tau)\left|\vartheta^{\prime}(z)\right|\right)\right] \mathrm{d} \tau \\
& +2 \alpha c(z-x) \int_{0}^{1}\left(\tau^{\alpha}-\tau^{\alpha+1}\right) \mathrm{d} \tau \\
= & \frac{z-x}{2}\left\{I_{1}+I_{2}+2 \alpha c(z-x) I_{3}\right\} .
\end{aligned}
$$

Integrating $I_{1}, I_{2}$, and $I_{3}$, we have

$$
\begin{aligned}
I_{1}= & \int_{0}^{1 / 2}\left((1-\tau)^{\alpha}-\tau^{\alpha}\right)\left[\left|\vartheta^{\prime}(m)\right|+\left|\vartheta^{\prime}(M)\right|-\left(\tau\left|\vartheta^{\prime}(x)\right|+(1-\tau)\left|\vartheta^{\prime}(z)\right|\right)\right] \mathrm{d} \tau \\
= & \left(\left|\vartheta^{\prime}(m)\right|+\left|\vartheta^{\prime}(M)\right|\right) \int_{0}^{1 / 2}\left((1-\tau)^{\alpha}-\tau^{\alpha}\right) \mathrm{d} \tau-\left\{\left|\vartheta^{\prime}(x)\right|\left[\int_{0}^{1 / 2} \tau(1-\tau)^{\alpha} \mathrm{d} \tau-\int_{0}^{1 / 2} \tau^{\alpha+1} \mathrm{~d} \tau\right]\right. \\
& \left.+\left|\vartheta^{\prime}(z)\right|\left[\int_{0}^{1 / 2}(1-\tau)^{\alpha+1} \mathrm{~d} \tau-\int_{0}^{1 / 2}(1-\tau) \tau^{\alpha} \mathrm{d} \tau\right]\right\} \\
= & \left(\left|\vartheta^{\prime}(m)\right|+\left|\vartheta^{\prime}(M)\right|\right)\left(\frac{1}{\alpha+1}-\frac{\left(1 / 2^{\alpha}\right)}{\alpha+1}\right)-\left\{\left|\vartheta^{\prime}(x)\right|\left(\frac{1}{(\alpha+1)(\alpha+2)}-\frac{\left(1 / 2^{\alpha+1}\right)}{\alpha+1}\right)\right. \\
& \left.+\left|\vartheta^{\prime}(z)\right|\left(\frac{1}{\alpha+2}-\frac{\left(1 / 2^{\alpha+1}\right)}{\alpha+1}\right)\right\}, \\
I_{2}= & \int_{1 / 2}^{1}\left(\tau^{\alpha}-(1-\tau)^{\alpha}\right)\left[\left|\vartheta^{\prime}(m)\right|+\left|\vartheta^{\prime}(M)\right|-\left(\tau\left|\vartheta^{\prime}(x)\right|+(1-\tau)\left|\vartheta^{\prime}(z)\right|\right)\right] \mathrm{d} \tau \\
= & \left(\left|\vartheta^{\prime}(m)\right|+\left|\vartheta^{\prime}(M)\right|\right) \int_{1 / 2}^{1}\left(\tau^{\alpha}-(1-\tau)^{\alpha}\right) \mathrm{d} \tau-\left\{\left|\vartheta^{\prime}(x)\right|\left[\int_{1 / 2}^{1} \tau^{\alpha+1} \mathrm{~d} \tau-\int_{1 / 2}^{1} \tau(1-\tau)^{\alpha} \mathrm{d} \tau\right]\right. \\
& \left.+\left|\vartheta^{\prime}(z)\right|\left[\int_{1 / 2}^{1}(1-\tau) \tau^{\alpha} \mathrm{d} \tau-\int_{1 / 2}^{1}(1-\tau)^{\alpha+1} \mathrm{~d} \tau\right]\right\} \\
I_{3}= & \frac{1}{(\alpha+1)(\alpha+2)} \cdot\left(\frac{1}{\alpha}-\frac{\left(1 / 2^{\alpha}\right)}{\alpha+1}\right)-\left\{\left|\vartheta^{\prime}(x)\right|\left(\frac{1}{\alpha+2}-\frac{\left(1 / 2^{\alpha+1}\right)}{\alpha+1}\right)\right. \\
= & \left.\left(\left|\vartheta^{\prime}(m)\right|+\left|\vartheta^{\prime}(M)\right|\right)\left(\frac{1}{\alpha+1}\right)\right\}, \\
& \left.+\left|\vartheta^{\prime}(z)\right|\left(\frac{1}{(\alpha+1)(\alpha+2)}-\frac{1}{\alpha+1}\right)\right\} \\
& \\
&
\end{aligned}
$$

Substituting the values of $I_{1}, I_{2}$, and $I_{3}$ in (48), we have 


$$
\begin{aligned}
& \mid \frac{\vartheta(m+M-x)+\vartheta(m+M-z)}{2}-\frac{\Gamma(\alpha+1)}{2(z-x)^{\alpha}}\left[\mathscr{f}_{(m+M-z)^{+}}^{\alpha} \mathcal{Y}(m+M-x)\right. \\
& \left.\quad+\mathscr{f}_{(m+M-x)^{-}}^{\alpha} \vartheta(m+M-z)\right]-\frac{\alpha c}{(\alpha+1)(\alpha+2)}(z-x)^{2} \mid \\
& \leq \frac{z-x}{2}\left\{2\left[\left|\vartheta^{\prime}(m)\right|+\left|\vartheta^{\prime}(M)\right|\right]\left[\frac{1}{\alpha+1}-\frac{1}{2^{\alpha}(\alpha+1)}\right]-\left|\vartheta^{\prime}(x)\right|\left[\frac{1}{(\alpha+1)(\alpha+2)}-\frac{1}{2^{\alpha+1}(\alpha+1)}\right]\right. \\
& \left.\quad-\left|\vartheta^{\prime}(z)\right|\left[\frac{1}{\alpha+2}-\frac{1}{2^{\alpha+1}(\alpha+1)}\right]-\left|\vartheta^{\prime}(z)\right|\left[\frac{1}{(\alpha+1)(\alpha+2)}-\frac{1}{2^{\alpha+1}(\alpha+1)}\right]+\frac{2 \alpha c(z-x)}{(\alpha+1)(\alpha+2)}\right\},
\end{aligned}
$$

and we obtain (47).

Theorem 10. Let $\vartheta:[m, M] \longrightarrow \mathbb{R}$ be a differentiable function, such that $\left|\vartheta^{\prime}\right|^{q}$ is convex on $[m, M]$, where $q>0$ and
$(1 / p)+(1 / q)=1$. Then, for $\alpha>0$ and for all $x, z \in[m, M]$, such that $x<z$ and, we have

$$
\begin{aligned}
& \mid \frac{\vartheta(m+M-x)+\vartheta(m+M-z)}{2}-\frac{\Gamma(\alpha+1)}{2(z-x)^{\alpha}}\left[\mathscr{J}_{(m+M-z)^{+}}^{\alpha}(m+M-x)\right. \\
& \left.\quad+\mathscr{J}_{(m+M-x)^{-}}^{\alpha} \vartheta(m+M-z)\right]-\frac{\alpha c}{(\alpha+1)(\alpha+2)}(z-x)^{2} \mid \\
& \leq \frac{z-x}{2}\left\{[L(\alpha, p)]^{1 / p}\left[\left|\vartheta^{\prime}(m)\right|^{q}+\left|\vartheta^{\prime}(M)\right|^{q}-\frac{\left|\vartheta^{\prime}(x)\right|^{q}+\left|\vartheta^{\prime}(z)\right|^{q}}{2}\right]^{1 / q}+\frac{2 \alpha c(z-x)}{(\alpha+1)(\alpha+2)}\right\},
\end{aligned}
$$

where $L(\alpha, p)=\int_{0}^{1}\left|\tau^{\alpha}-(1-\tau)^{\alpha}\right|^{p} d \tau$.

Proof. Using Lemma 1 and applying Hölder's inequality, we have

$$
\begin{aligned}
& \mid \frac{\vartheta(m+M-x)+\vartheta(m+M-z)}{2}-\frac{\Gamma(\alpha+1)}{2(z-x)^{\alpha}}\left[\mathscr{F}_{(m+M-z)^{+}}^{\alpha} \vartheta(m+M-x)\right. \\
& \left.\quad+\mathscr{J}_{(m+M-x)^{-}}^{\alpha} \vartheta(m+M-z)\right]-\frac{\alpha c}{(\alpha+1)(\alpha+2)}(z-x)^{2} \mid \\
& \leq \frac{z-x}{2}\left\{\left[\int_{0}^{1}\left|\tau^{\alpha}-(1-\tau)^{\alpha}\right|^{p} \mathrm{~d} \tau\right]^{1 / p}\left[\int_{0}^{1}\left|\vartheta^{\prime}(m+M-(\tau x+(1-\tau) z))\right|^{q} \mathrm{~d} \tau\right]^{1 / q}\right. \\
& \left.\quad+2 \alpha c(z-x) \int_{0}^{1}\left|\tau^{\alpha}-\tau^{\alpha+1}\right| \mathrm{d} \tau\right\} .
\end{aligned}
$$

Using the convexity of $\left|\vartheta^{\prime}\right|^{q}$ and the Jensen-Mercer inequality, we get 


$$
\begin{aligned}
& \mid \frac{\vartheta(m+M-x)+\vartheta(m+M-z)}{2}-\frac{\Gamma(\alpha+1)}{2(z-x)^{\alpha}}\left[\mathscr{J}_{(m+M-z)^{+}}^{\alpha} \vartheta(m+M-x)\right. \\
& \left.\quad+\mathscr{J}_{(m+M-x)^{-}}^{\alpha} \vartheta(m+M-z)\right]-\frac{\alpha c}{(\alpha+1)(\alpha+2)}(z-x)^{2} \mid \\
& \leq \frac{z-x}{2}\left\{[ L ( \alpha , p ) ] ^ { 1 / p } \left[\int _ { 0 } ^ { 1 } \left(\left|\vartheta^{\prime}(m)\right|^{q}+\left|\vartheta^{\prime}(M)\right|^{q}\right.\right.\right. \\
& \left.\left.\left.\quad-\left(\tau\left|\vartheta^{\prime}(x)\right|^{q}+(1-\tau)\left|\vartheta^{\prime}(z)\right|^{q}\right)\right) \mathrm{d} \tau\right]^{1 / q}+\frac{2 \alpha c(z-x)}{(\alpha+1)(\alpha+2)}\right\},
\end{aligned}
$$

and (51) is immediate.

$$
\begin{aligned}
& \mid \frac{2^{\alpha-1} \Gamma(\alpha+1)}{(z-x)^{\alpha}}\left[\mathscr{J}_{(m+M-((x+z) / 2))^{+}}^{\alpha} \vartheta(m+M-x)+\mathscr{J}_{(m+M-((x+z) / 2))^{-}}^{\alpha} \vartheta(m+M-z)\right] \\
& \quad-\vartheta\left(m+M-\frac{x+z}{2}\right)-\frac{c}{2(\alpha+1)(\alpha+2)}(z-x)^{2} \mid \\
& \leq \frac{z-x}{2(\alpha+1)}\left\{\left|\vartheta^{\prime}(m)\right|+\left|\vartheta^{\prime}(M)\right|-\frac{\left|\vartheta^{\prime}(x)\right|+\left|\vartheta^{\prime}(z)\right|}{2}+\frac{c}{\alpha+2}(z-x)\right\} .
\end{aligned}
$$

Proof. From Lemma 2, we can write

$$
\begin{aligned}
& \mid \frac{2^{\alpha-1} \Gamma(\alpha+1)}{(z-x)^{\alpha}}\left[\mathscr{J}_{(m+M-((x+z) / 2))^{+}}^{\alpha} \vartheta(m+M-x)+\mathscr{J}_{(m+M-((x+z) / 2))^{-}}^{\alpha} \vartheta(m+M-z)\right] \\
& \quad-\vartheta\left(m+M-\frac{x+z}{2}\right)-\frac{c}{2(\alpha+1)(\alpha+2)}(z-x)^{2} \mid \\
& \leq \frac{z-x}{4}\left\{\int_{0}^{1} \tau^{\alpha}\left|\vartheta^{\prime}\left(m+M-\left(\frac{2-\tau}{2} x+\frac{\tau}{2} z\right)\right)\right| \mathrm{d} \tau+\int_{0}^{1} \tau^{\alpha}\left|\vartheta^{\prime}\left(m+M-\left(\frac{\tau}{2} x+\frac{2-\tau}{2} z\right)\right)\right| \mathrm{d} \tau\right. \\
& \left.\quad+2 c(z-x) \int_{0}^{1} \tau^{\alpha}|1-\tau| \mathrm{d} \tau\right\} .
\end{aligned}
$$

Using the convexity of $\left|\vartheta^{\prime}\right|$, we have

$$
\begin{aligned}
& \mid \frac{2^{\alpha-1} \Gamma(\alpha+1)}{(z-x)^{\alpha}}\left[\mathcal{F}_{(m+M-((x+z) / 2))^{+}}^{\alpha}(m+M-x)+\mathscr{J}_{(m+M-((x+z) / 2))^{-}}^{\alpha} \vartheta(m+M-z)\right] \\
& \quad-\vartheta\left(m+M-\frac{x+z}{2}\right)-\frac{c}{2(\alpha+1)(\alpha+2)}(z-x)^{2} \mid \\
& \leq \frac{z-x}{4}\left\{\left[\left|\vartheta^{\prime}(m)\right|+\left|\vartheta^{\prime}(M)\right|\right] \int_{0}^{1} \tau^{\alpha} \mathrm{d} \tau-\int_{0}^{1} \tau^{\alpha}\left(\frac{2-\tau}{2}\left|\vartheta^{\prime}(x)\right|+\frac{\tau}{2}\left|\vartheta^{\prime}(z)\right|\right) \mathrm{d} \tau+\left[\left|\vartheta^{\prime}(m)\right|+\left|\vartheta^{\prime}(M)\right|\right]\right.
\end{aligned}
$$




$$
\begin{aligned}
& \left.\int_{0}^{1} \tau^{\alpha} \mathrm{d} \tau-\int_{0}^{1} \tau^{\alpha}\left(\frac{\tau}{2}\left|\vartheta^{\prime}(x)\right|+\frac{2-\tau}{2}\left|\vartheta^{\prime}(z)\right|\right) \mathrm{d} \tau+2 c(z-x)\left[\int_{0}^{1} \tau^{\alpha} \mathrm{d} \tau-\int_{0}^{1} \tau^{\alpha+1} \mathrm{~d} \tau\right]\right\} \\
& =\frac{z-x}{4}\left\{\frac{2\left[\left|\vartheta^{\prime}(m)\right|+\left|\vartheta^{\prime}(M)\right|\right]}{\alpha+1}-\frac{\left|\vartheta^{\prime}(x)\right|(\alpha+3)}{2(\alpha+1)(\alpha+2)}-\frac{\left|\vartheta^{\prime}(z)\right|}{2(\alpha+2)}\right. \\
& \left.\quad-\frac{\left|\vartheta^{\prime}(x)\right|}{2(\alpha+2)}-\frac{\left|\vartheta^{\prime}(z)\right|(\alpha+3)}{2(\alpha+1)(\alpha+2)}+\frac{2 c(z-x)}{(\alpha+1)(\alpha+2)}\right\}
\end{aligned}
$$

and we get (54).

Theorem 12. Let all the assumptions of Theorem 10 hold. Then,

$$
\begin{aligned}
& \mid \frac{2^{\alpha-1} \Gamma(\alpha+1)}{(z-x)^{\alpha}}\left[\mathscr{J}_{(m+M-((x+z) / 2))^{+}}^{\alpha} \vartheta(m+M-x)+\mathscr{J}_{(m+M-((x+z) / 2))^{-}}^{\alpha} \vartheta(m+M-z)\right] \\
& \quad-\vartheta\left(m+M-\frac{x+z}{2}\right)-\frac{c}{2(\alpha+1)(\alpha+2)}(z-x)^{2} \mid \\
& \leq \frac{z-x}{4}\left(\frac{1}{\alpha p+1}\right)^{1 / p}\left\{\left[\left|\vartheta^{\prime}(m)\right|^{q}+\left|\vartheta^{\prime}(M)\right|^{q}-\frac{3\left|\vartheta^{\prime}(x)\right|^{q}+\left|\vartheta^{\prime}(z)\right|^{q}}{4}\right]^{1 / q}\right. \\
& \left.\quad+\left[\left|\vartheta^{\prime}(m)\right|^{q}+\left|\vartheta^{\prime}(M)\right|^{q}-\frac{\left|\vartheta^{\prime}(x)\right|^{q}+3\left|\vartheta^{\prime}(z)\right|^{q}}{4}\right]^{1 / q}+\frac{2 c(z-x)}{(q+1)^{1 / q}}\right\} .
\end{aligned}
$$

Proof. By using Lemma 2 and Hölder's inequality, we can write

$$
\begin{aligned}
& \mid \frac{2^{\alpha-1} \Gamma(\alpha+1)}{(z-x)^{\alpha}}\left[\mathscr{J}_{(m+M-((x+z) / 2))^{+}}^{\alpha} \vartheta(m+M-x)+\mathscr{J}_{(m+M-((x+z) / 2))^{-}}^{\alpha} \vartheta(m+M-z)\right] \\
& \quad-\vartheta\left(m+M-\frac{x+z}{2}\right)-\frac{c}{2(\alpha+1)(\alpha+2)}(z-x)^{2} \mid \\
& \leq \frac{z-x}{4}\left(\int_{0}^{1} \tau^{\alpha p} \mathrm{~d} \tau\right)^{1 / p}\left\{\left[\int_{0}^{1}\left|\vartheta^{\prime}\left(m+M-\left(\frac{2-\tau}{2} x+\frac{\tau}{2} z\right)\right)\right|^{q} \mathrm{~d} \tau\right]^{1 / q}\right. \\
& \left.\quad+\left[\int_{0}^{1}\left|\vartheta^{\prime}\left(m+M-\left(\frac{\tau}{2} x+\frac{2-\tau}{2} z\right)\right)\right|^{q} \mathrm{~d} \tau\right]^{1 / q}+2 c(z-x)\left[\int_{0}^{1}|1-\tau|^{q} \mathrm{~d} \tau\right]^{1 / q}\right\} .
\end{aligned}
$$

Using the convexity of $\left|\vartheta^{\prime}\right|^{q}$ and simplifying, we obtain

$$
\begin{aligned}
& \mid \frac{2^{\alpha-1} \Gamma(\alpha+1)}{(z-x)^{\alpha}}\left[\mathscr{J}_{(m+M-((x+z) / 2))^{+}}^{\alpha} \vartheta(m+M-x)+\mathscr{J}_{(m+M-((x+z) / 2))^{-}}^{\alpha} \vartheta(m+M-z)\right] \\
& \quad-\vartheta\left(m+M-\frac{x+z}{2}\right)-\frac{c}{2(\alpha+1)(\alpha+2)}(z-x)^{2} \mid
\end{aligned}
$$




$$
\begin{aligned}
& \leq \frac{z-x}{4}\left(\int_{0}^{1} \tau^{\alpha p} \mathrm{~d} \tau\right)^{1 / p}\left\{\left[\left[\left|\vartheta^{\prime}(m)\right|^{q}+\left|\vartheta^{\prime}(M)\right|^{q}\right] \int_{0}^{1} \mathrm{~d} \tau-\int_{0}^{1}\left(\frac{2-\tau}{2}\left|\vartheta^{\prime}(x)\right|^{q}+\frac{\tau}{2}\left|\vartheta^{\prime}(z)\right|^{q}\right) \mathrm{d} \tau\right]^{1 / q}\right. \\
& \quad+\left[\left[\left|\vartheta^{\prime}(m)\right|^{q}+\left|\vartheta^{\prime}(M)\right|^{q}\right] \int_{0}^{1} \mathrm{~d} \tau-\int_{0}^{1}\left(\frac{\tau}{2}\left|\vartheta^{\prime}(x)\right|^{q}+\frac{2-\tau}{2}\left|\vartheta^{\prime}(z)\right|^{q}\right) \mathrm{d} \tau\right]^{1 / q} \\
& \left.\quad+2 c(z-x)\left[\int_{0}^{1}(1-\tau)^{q} \mathrm{~d} \tau\right]^{1 / q}\right\},
\end{aligned}
$$

and (57) is immediate.

\section{Data Availability}

No data were used to support this study.

\section{Conflicts of Interest}

The authors declare that there are no conflicts of interest.

\section{Authors' Contributions}

All authors contributed equally to writing of this manuscript. All authors read and approved the final manuscript.

\section{References}

[1] M. J. Cloud, B. C. Drachman, and L. P. Lebedev, Inequalities with Applications to Engineering, Springer, London, UK, 2014.

[2] J. G. Liao and A. Berg, "Sharpening Jensen's inequality," The American Statistician, vol. 73, no. 1, 2018.

[3] General Inequalities 7, "General inequalities 7," Journal of Approximation Theory, vol. 94, no. 3, pp. 507-508, 1998.

[4] S. Fridli and F. Schipp, "Strong approximation via Sidon type inequalities," Journal of Approximation Theory, vol. 94, no. 2, pp. 263-284, 1998.

[5] S. Sever Dragomir, M. Adil Khan, and A. Abathun, "Refinement of the Jensen integral inequality," Open Mathematics, vol. 14, no. 1, pp. 221-228, 2016.

[6] S. S. Dragomir, "Inequalities with applications in numerical analysis," in AIP Conference Proceedings, vol. 936, no. 1, p. 681, American Institute of Physics, 2007.

[7] B. T. Polyak, "Existence theorems and convergence of minimizing sequences for extremal problems with constraints," in Doklady Akademii Naukvol. 166, no. 2, pp. 287-290, Russian Academy of Sciences, 1966.

[8] K. Nikodem, "On strongly convex functions and related classes of functions," in Handbook of Functional Equations, pp. 365-405, Springer, New York, NY, USA, 2014.

[9] K. Nikodem and Z. Pales, "Characterizations of inner product spaces by strongly convex functions," Banach Journal of Mathematical Analysis, vol. 5, no. 1, pp. 83-87, 2011.

[10] E. S. Polovinkin, "On strongly convex sets and strongly convex functions," Journal of Mathematical Sciences, vol. 100, no. 6, pp. 2633-2681, 2000.

[11] S. S. Dragomir and C. Pearce, "Selected topics on HermiteHadamard inequalities and applications," Science Direct, pp. S1574-S2358, 2003, Working Paper.

[12] N. Merentes and K. Nikodem, "Remarks on strongly convex functions," Aequationes Mathematicae, vol. 80, no. 1-2, pp. 193-199, 2010.
[13] M. Adil Khan, T. Ali, and T. U. Khan, "Hermite-Hadamard type inequalities with applications," Fasciculi Mathematici, vol. 59, no. 1, pp. 57-74, 2017.

[14] J. Wang, X. Li, and Y. Zhou, "Hermite-Hadamard inequalities involving Riemann-Liouville fractional integrals via s-convex functions and applications to special means," Filomat, vol. 30, no. 5, pp. 1143-1150, 2016.

[15] T. U. Khan and M. Adil Khan, "Hermite-Hadamard inequality for new generalized conformable fractional operators," AIMS Math, vol. 6, pp. 23-38, 2020.

[16] Y. Khurshid, M. Adil Khan, and Y.-M. Chu, "Conformable integral version of Hermite-Hadamard-Fejér inequalities via $\eta$-convex functions," AIMS Mathematics, vol. 5, no. 5, pp. 5106-5120, 2020.

[17] S. S. Dragomir, "Inequalities of Hermite-Hadamard type for HG-convex functions," Issues of Analysis, vol. 6, no. 2, 2017.

[18] E. Set, J. Choi, and A. Gözpinar, "Hermite-Hadamard type inequalities for the generalized $k$-fractional integral operators," Journal of Inequalities and Applications, vol. 2017, no. 1, pp. 1-17, 2017.

[19] E. Set, İ. İşcan, M. Zeki Sarikaya, and M. Emin Özdemir, “On new inequalities of Hermite-Hadamard-Fejér type for convex functions via fractional integrals," Applied Mathematics and Computation, vol. 259, pp. 875-881, 2015.

[20] E. Set and M. Tomar, "New inequalities of Hermite-Hadamard type for generalized convex functions with applications," Facta Universitatis-Series: Mathematics and Informatics, vol. 31, no. 2, pp. 383-397, 2016.

[21] M. Z. Sarikaya, E. Set, H. Yaldiz, and N. Basak, "HermiteHadamards inequalities for fractional integrals and related fractional inequalities," Mathematical and Computer Modelling, vol. 57, no. 9-10, pp. 2403-2407, 2013.

[22] A. M. Mercer, "A variant of Jensens inequality," Journal of Inequalities in Pure and Applied Mathematics, vol. 6, no. 4, p. 73, 2003.

[23] H. Öğülmüş and M. Z. Sarikaya, "Hermite-Hadamard-Mercer type inequalities for fractional integrals," Researchgate, 2020.

[24] H. Wang, J. Khan, M. Adil Khan, S. Khalid, and R. Khan, "The Hermite-Hadamard-Jensen-Mercer type inequalities for Riemann-Liouville fractional integral," Journal of Mathematics, vol. 2021, Article ID 5516987, 18 pages, 2021.

[25] S. I. Butt, M. Umar, S. Rashid, A. O. Akdemir, and Y. M. Chu, "New Hermite-Jensen-Mercer-type inequalities via $\mathrm{k}$-fractional integrals," Advances in Difference Equations, vol. 2020, no. 1, pp. 1-24, 2020.

[26] S. I. Butt, M. Nadeem, S. Qaisar, A. O. Akdemir, and T. Abdeljawad, "Hermite-Jensen-Mercer type inequalities for conformable integrals and related results," Advances in Difference Equations, vol. 2020, no. 1, pp. 1-24, 2020.

[27] M. Vivas-Cortez, M. A. Ali, M. A. Ali, A. Kashuri, and H. Budak, "Generalizations of fractional Hermite-Hadamard-Mercer like 
inequalities for convex functions," AIMS Mathematics, vol. 6, no. 9, pp. 9397-9421, 2021.

[28] H. R. Moradi, M. E. Omidvar, M. Adil Khan, and K. Nikodem, "Around Jensen's inequality for strongly convex functions," Aequationes Mathematicae, vol. 92, no. 1, pp. 25-37, 2018.

[29] A. Matkovic and J. Pecaric, "On a variant of the JensenMercer inequality for operators," Journal of Mathematical Inequalities, vol. 2, no. 3, pp. 299-307, 2008.

[30] M. Kian and M. Moslehian, "Refinements of the operator Jensen-Mercer inequality," The Electronic Journal of Linear Algebra, vol. 26, pp. 742-753, 2013.

[31] M. Adil Khan, G. Ali Khan, M. Jameel, K. A. Khan, and A. Kilicman, "New refinements of Jensen-Mercer's inequality," Journal of Computational and Theoretical Nanoscience, vol. 12, no. 11, pp. 4442-4449, 2015.

[32] A. S. Vatsala and V. Lakshmikantham, "Fractional differential and integral equations of Riemann-Liouville versus caputo," in AIP Conference Proceedings, vol. 1067, no. 1, pp. 87-93, American Institute of Physics, 2008.

[33] G. Farid, "Some Riemann-Liouville fractional integral inequalities for convex functions," The Journal of Analysis, vol. 27, no. 4, pp. 1095-1102, 2019. 